Dept. of Math. Univ. of Oslo

Statistical Research Report No. 5

ISSN 0806-3842 May 2005

\title{
A SPATIAL-TEMPORAL MODEL FOR TEMPERATURE WITH SEASONAL VARIANCE
}

\author{
FRED ESPEN BENTH, JŪRATE் ŠALTYTĖ-BENTH, AND PAULIUS JALINSKAS
}

\begin{abstract}
We propose a spatial-temporal stochastic model for daily average temperature data. First we build a model for a single spatial location, independently on the spatial information. The model includes trend, seasonality and mean-reversion, together with a seasonally dependent variance of the residuals. The spatial dependency is modelled by a Gaussian random field. Empirical fitting to data collected in 16 measurement stations in Lithuania over more than 40 years shows that our model captures the seasonality in the autocorrelation of the squared residuals, a property of temperature data already observed by other authors. We demonstrate with some examples that our spatial-temporal model is applicable for prediction and classification.
\end{abstract}

\section{INTRODUCTION}

Temperature is an important characteristic of the thermodynamical state of the atmosphere, and probably the most important element since it controls or influences other elements like, for example humidity, clouds, air pressure and precipitation. In this paper we propose a spatial-temporal Gaussian random field for modelling the daily average temperature (DAT), and fit this to data collected in Lithuania.

The changes of the spatial distribution of temperature is the result of global and local factors. One such factor is the inflow of solar radiation, which, generally depends on the geographical location. In Lithuania the location of seas and continents - Baltic Sea, Scandinavian Peninsula and Atlantic Ocean to the West of Lithuania and large Eurasia continent to the East of the country determine differences between coastal and inland localities. The coastal locations experience moist Baltic Sea air most of the year, while moving to the East, temperature is influenced by the more continental atmosphere circulation regimes. Different scales of atmosphere circulation - predominant Westerly circulation in the midlatitudes is next important factor driven by temperature and pressure differences between warm/low and cold/high latitudes together with the Coriolis Effect. Other factors are types of surface layer and local relief. Seasonal fluctuations of these factors determine the characteristics of the temperature distribution. During the year characteristics and domination of factors may vary which can be seen on spatial distribution of the isotherms.

Date: May 30, 2005.

Key words and phrases. Spatial-temporal random field, daily average temperature, seasonally dependent variance.

We are grateful to the Lithuanian Hydrometeorological Service for providing us with data. Jūratè Šaltytė-Benth is supported by the Norwegian Research Council under grant NFR: 155120/432. 
We propose to model the DAT by a Gaussian random field which is seasonally meanreverting in time and having a seasonal variance. Campbell and Diebold (2005) observed that in several American cities squaring the residuals obtained after removing the trend, seasonality and mean-reversion possessed a seasonal autocorrelation function. The same phenomena was (more or less clearly) observed for Norwegian data and Stockholm in the papers Benth and Šaltytė-Benth (2005a,b), where the authors suggested to model this by a deterministic seasonal variance function. Campbell and Diebold (2005) model the DAT by an autoregressive time series with a seasonal ARCH-type dynamics for the residuals. In the present paper we extend the simple time series model in Benth and Šaltytė-Benth (2005a,b) to a spatial-temporal model that is consistent with the time series model for each location, but taking into account the spatial correlation between stations. We demonstrate that our model is simple, but yet powerful enough to model the most important statistical properties of the temperature dynamics.

We consider two examples involving temperature prediction and classification, demonstrating that our model is analytically tractable for natural applications. The question of future temperature prediction is important given a statistical model, and we apply kriging techniques in order to predict the day-ahead temperature together with the error in a city which is not included in the data set used for estimating the model. Since temperature is part of a set of variables used to classify weather and climate regions, one may ask how discriminant analysis can be applied to our model. We show that it is indeed possible to set up a classification scheme, and demonstrate this with considering the question of assigning a station to a climate region solely on temperature observations.

Many papers dealing with temperature modelling have analysis of financial weather derivatives in mind as the prime application. Campbell and Diebold (2005) argue for their model in light of such applications, and the motivation of Benth and Saltytė-Benth $(2005 \mathrm{a}, \mathrm{b})$ is to price financial weather contracts. In order to do so successfully, one needs to have a model that is easily formulated in a continuous-time set-up, which will be the case for our suggested temperature dynamical model. In the weather derivatives markets there are investors who issue (or buy) contracts based on temperature in many locations, and the spatial dependency structure then becomes important. Furthermore, one may think of an investor whose profit is highly dependent on temperature at a location for which there are no derivatives traded (e.g. a ski resort). This investor will benefit from a spatial model to be able to see how his location is dependent on a location for which there exists official and trustable measurements of temperature, and where derivatives may be issued. This is a financial application of our spatial-temporal temperature model. Among several papers dealing with temperature derivatives, we would like to mention Alaton, Djehiche and Stillberger (2002) who analyze a Swedish temperature series using a mean-reverting model having monthly variability in the residuals. Brody, Syroka and Zervos (2002) analyze a mean-reverting fractional Brownian motion to model temperature.

The rest of the paper is divided into 3 sections. The next one contains a detailed description of the temperature data collected in Lithuania. Here we perform a simple statistical analysis to get more feeling about such features as normality, seasonality and 
other properties. This helps us to do some considerations about the possible model. We present the model in the first part of the third section. Later we fit the suggested model to our data. Analysis of residuals shows that the proposed model captures the properties of data quite well. Here we also perform a validation of our model. In the last section we demonstrate with some examples how our model can be applied to prediction and classification.

\section{The DATA}

We start with a description of the collection of temperature data. There are 20 meteorological stations in Lithuania (see Fig. 1), where temperatures are measured every 3 hours from 00:00 to 21:00 UTC in degrees of Celsius. Our objective in this paper is DAT, which is obtained by averaging the 8 values of temperature observed a certain day at each station. The data base is available through the Lithuanian Hydrometeorological Service in Vilnius, Lithuania.

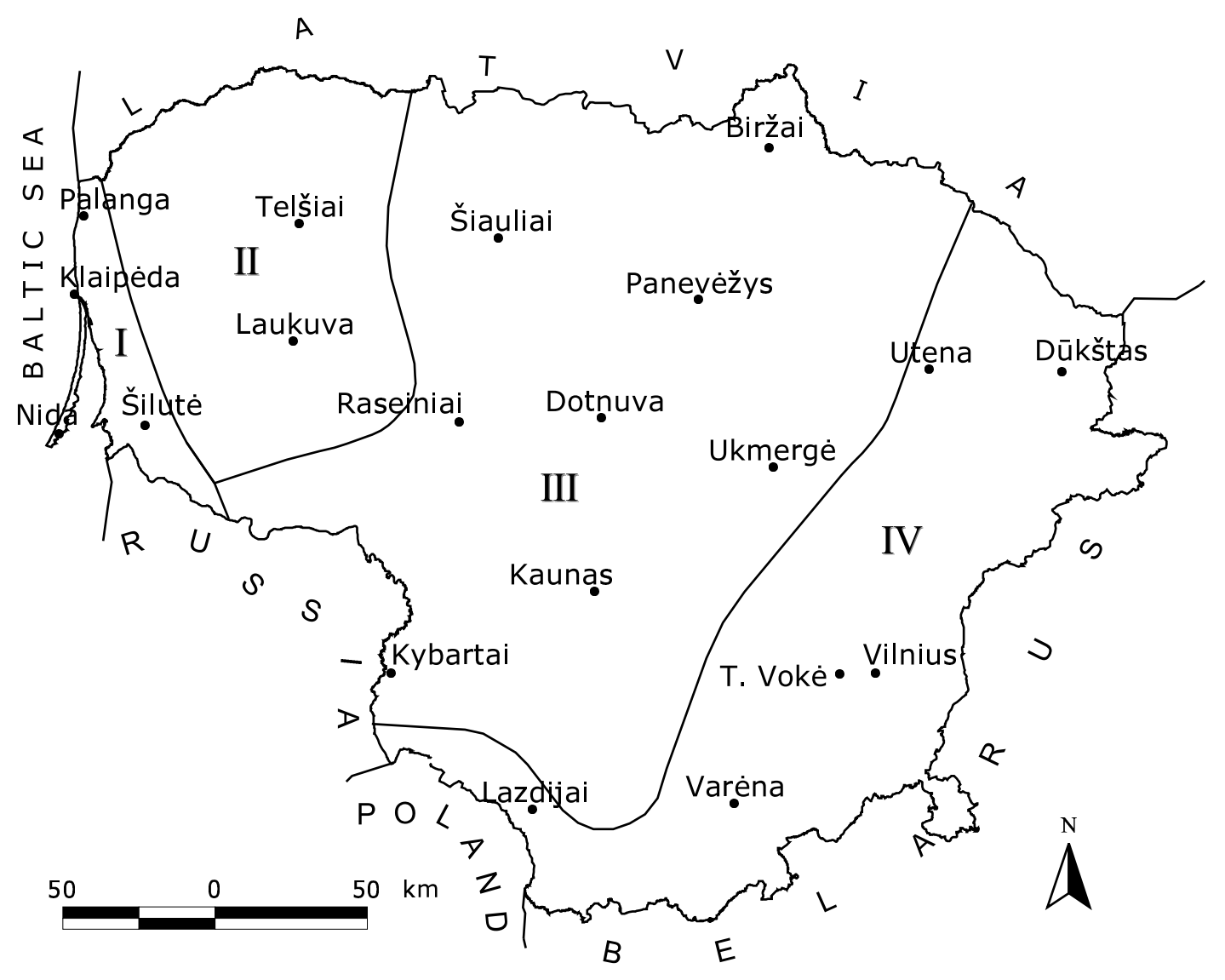

FiguRE 1. Map of Lithuania with measurement stations and climate regions marked with Roman numerals. T.Vokè is abbreviation for Traku̧ Vokè. 
In most of the stations we have observations in electronic form starting in the beginning of 1961, except for Vilnius where the starting point is the beginning of June 1964. We exclude 4 measurement stations (Dūkštas, Lazdijai, Palanga and Traku̧ Vokè) from the analysis because of too many missing observations. There were some missing observations in other stations as well, which we filled in (for each station separately) by the average of observations made at the same day in the last 5 years.

In order to have time series of equal length in all 16 stations, as a starting point we choose the 1st of June 1964. DAT are ranging until the 31 of August 2004 resulting in 14692 observations. When illustrating our results graphically, we choose only 4 stations: Klaipeda, Telšiai, Dotnuva and Vilnius. The choice of these stations was based on the climate map of Lithuania, where there are 4 regions, and the 4 chosen stations are representatives of these climate regions. In Fig. 2 we plot the DAT from the beginning of 2001 (3 years and 8 month), as the pattern for other time periods is basically the same.

Before proceeding to the modelling of DAT, we give some simple descriptive statistics characteristics for all 16 stations, that are resumed in Table 1 . The variation in different characteristics of DAT is not big among the stations. The median DAT and the average DAT are somewhat different from each other, which may indicate a deviation from the normality in data. The values of skewness and kurtosis are also supporting the hypothesis of non-normality in DAT: both they are small, but still different from zero. Note that all values of skewness and kurtosis are negative, indicating left skewness and a less peaky distribution than the normal.

Analysis of histograms of all stations confirms the conclusions reached above. All 16 histograms have similar shape. In Fig. 3 we present the histograms of the 4 representative stations. All they have left skewness and have no clear peak which is contradictory to the normality. In fact, the distributions seem to be bimodal, which can be explained by different seasons of cool and warm weather. We performed a correlation analysis between stations, where correlations for each pair of time series were calculated. All correlations are very close to +1 and (as expected) depend on distance between stations: observations in stations far apart are less correlated than in those within shorter distances. High values of the correlations can be explained by fact that stations are relatively close to each other (the longest distance between two stations being approximately $292 \mathrm{~km}$ ) and temperatures in Lithuania vary in a quite similar manner on long time scales.

Values of the Kolmogorov-Smirnov statistics were statistically significant for all 16 measurement stations at the $1 \%$ level, which gives us a strong evidence of non-normality in DAT. To be confident in the choice of the model, we also check for the presence of autocorrelation in DAT. From Fig. 4 we see a strong seasonal variation in the values of autocorrelations, indicating seasonal heteroskedasticity in data.

Before defining our spatial-temporal Gaussian random field, let us briefly describe the modelling idea in connection to the DAT observations. In view of the many discussions of a global warming (see e.g., Rassmusson et al. (1993) or Handcock and Wallis (1994)), a first step in the data analysis would be to check for the presence of a linear trend. Next, we investigate the seasonal pattern of the DAT. Already from Fig. 2 it is obvious that 

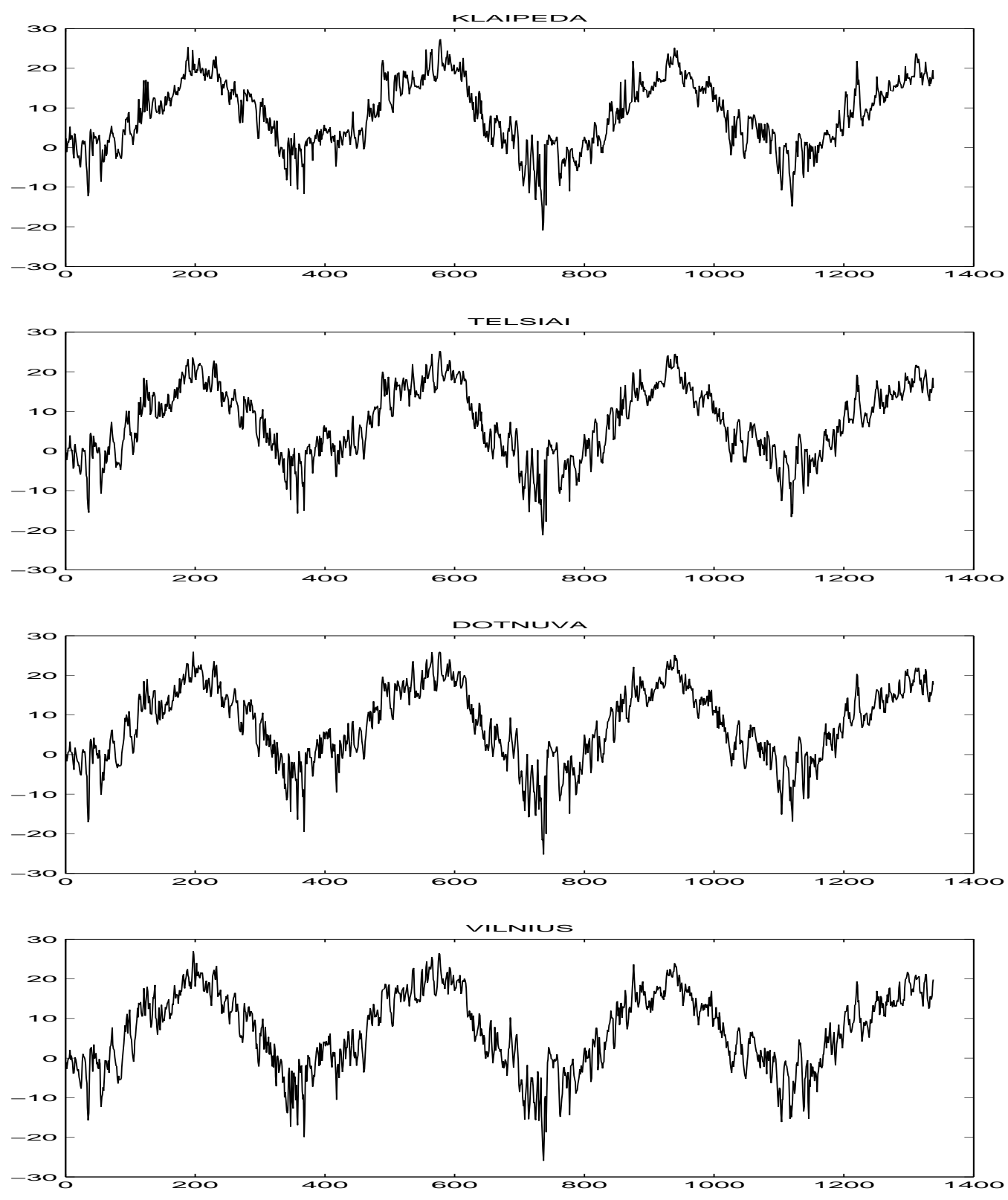

Figure 2. DAT in Klaipėda, Telšiai, Dotnuva and Vilnius in the period 20010101 to 20040831.

temperatures vary with season, being low in the winter and high in the summer. We model these seasonal variations with a simple cosine function.

Due to natural cyclic/periodic temperature variations, which are seen on diurnal, interseasonal or annual time scales, temperature has a tendency to revert back to its mean over 

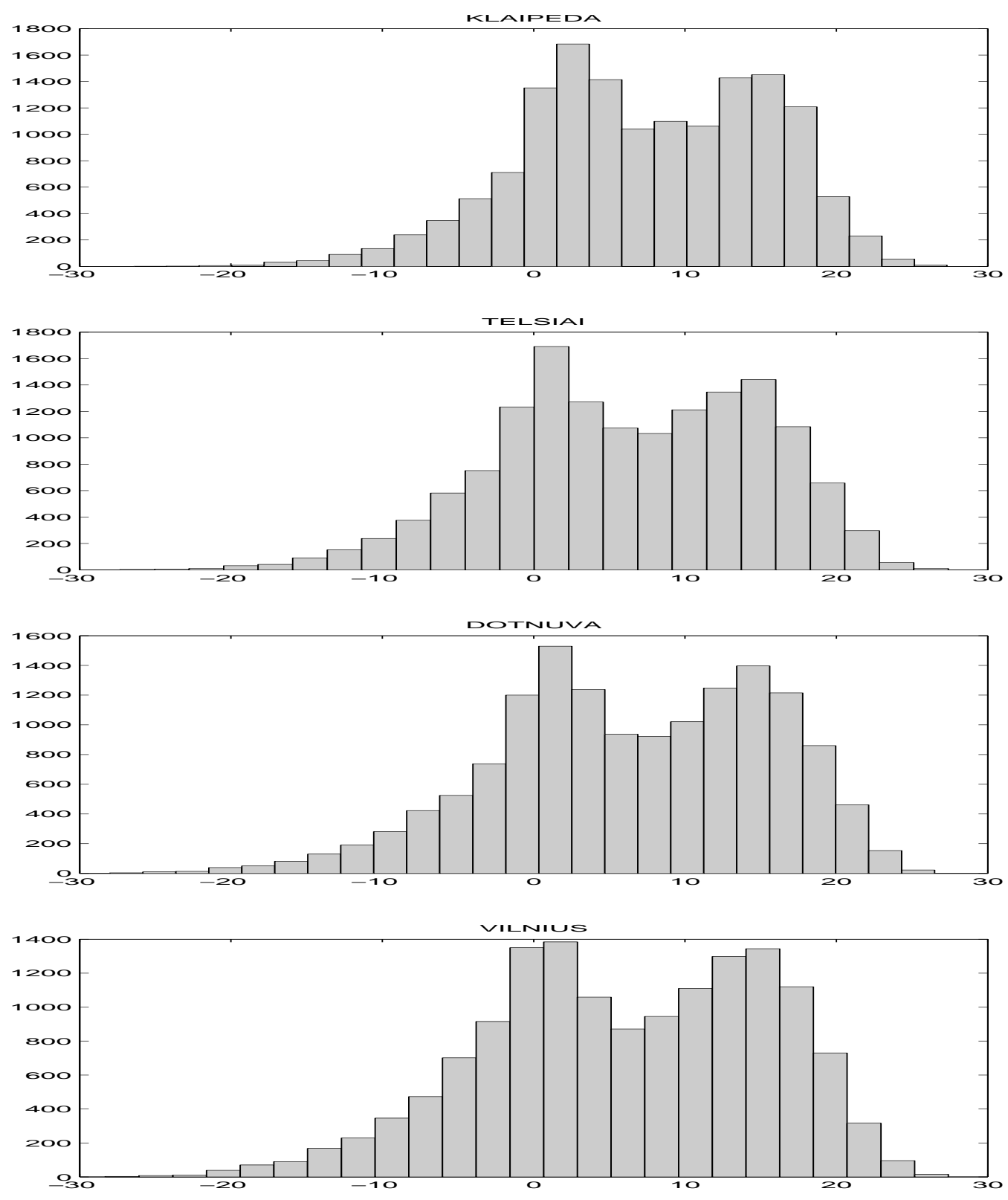

Figure 3. Histograms of DAT in Klaipèda, Telšiai, Dotnuva and Vilnius.

time. This mean-reverting property is modelled by a first order autoregressive (AR(1)) process.

We eliminate step by step the different modelling components from the data. First, we eliminate the linear trend, then we deseasonalize data and apply $\mathrm{AR}(1)$ for the resulting data. We show that the obtained residuals in the AR(1) model are not uncorrelated and far from being normal. The autocorrelation function for squared residuals reveals the presence 

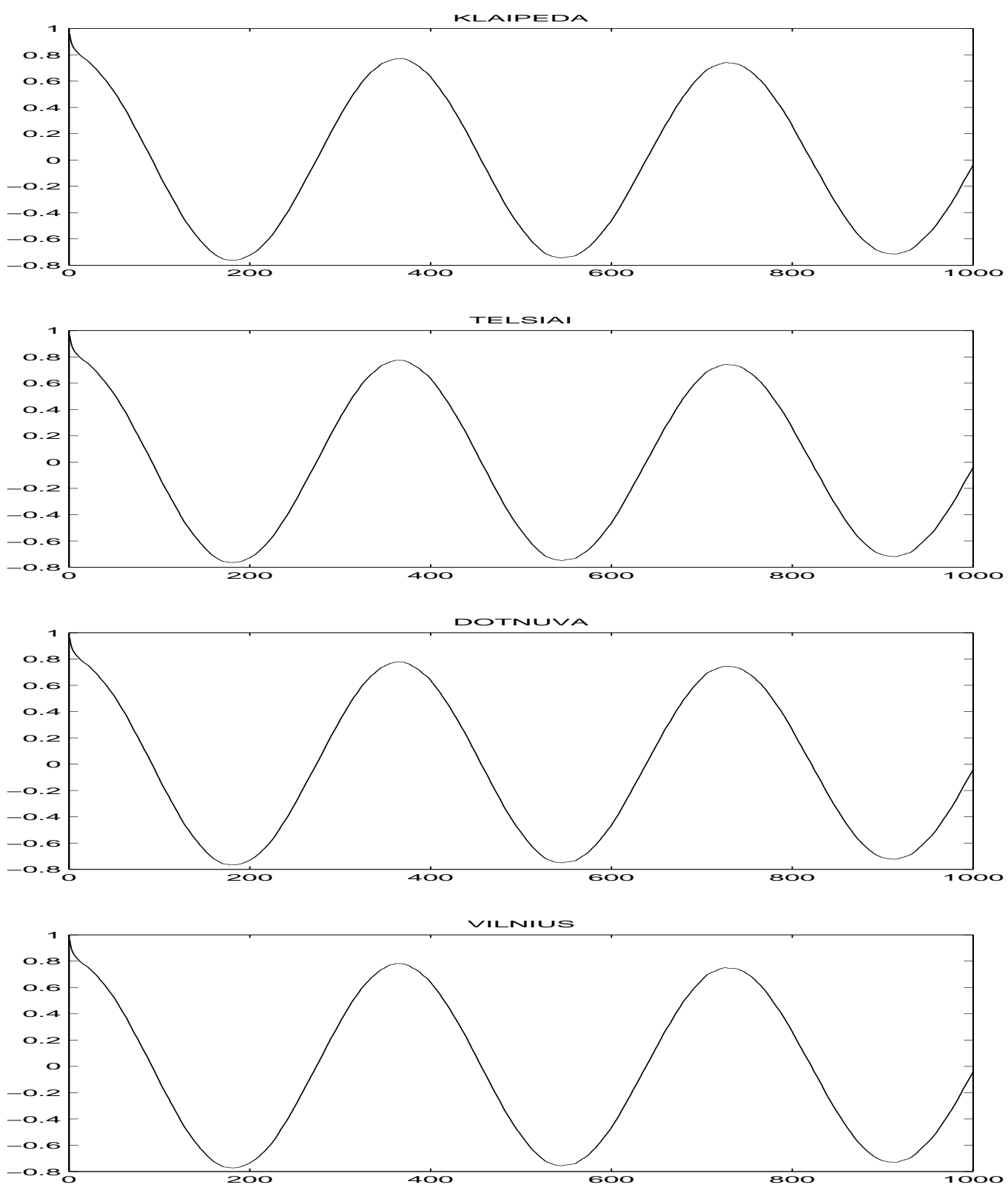

Figure 4. Autocorrelation functions of DAT in Klaipėda, Telšiai, Dotnuva and Vilnius.

of a seasonally dependent variance, which we explain using a truncated Fourier series. After we model daily seasonal variance and remove its effect from data, the residuals become close to normal, being only slightly correlated for the few first lags, and uncorrelated when the number of lags increases. 
After fitting the process at each location separately as a time series, we turn our attention to the spatial modelling. We suppose that the residuals come from a Gaussian random field and fit the spatial correlation function based on the empirical correlations between locations.

\section{MODEL}

In this section we define the spatial-temporal Gaussian random field that we propose for modelling the temperature evolution in time and space. After doing this we fit the model to the DAT observations we have available for Lithuania, and finally validate our model by comparing with observations from locations not included in the estimation procedure.

3.1. Model specification. Spatial-temporal data can be considered as a realization of a random field

$$
\left\{Z(\mathbf{s} ; t): \mathbf{s} \in D \subset R^{2}, t \in[0, \infty)\right\}
$$

where $\mathbf{s}$ and $t$ define spatial and temporal coordinates, respectively.

Typically, a spatial-temporal model $Z(\mathbf{s} ; t)$ is decomposed into a mean component $\mu(\mathbf{s} ; t)$ modelling the trend, and residual component $\varepsilon(\mathbf{s} ; t)$ modelling the fluctuations around the trend in both space and time. Hence, the decomposition of the spatial-temporal random field $Z(\mathbf{s} ; t)$ can be written as

$$
Z(\mathbf{s} ; t)=\mu(\mathbf{s} ; t)+\varepsilon(\mathbf{s} ; t)
$$

where $\mu(\mathbf{s} ; t)$ is a deterministic function of the space-time coordinates. We suppose it to be given by

$$
\mu(\mathbf{s} ; t)=S(\mathbf{s} ; t)+\alpha(\mathbf{s})(Z(\mathbf{s} ; t-1)-S(\mathbf{s} ; t-1))
$$

where the mean-reversion parameter $\alpha(\mathbf{s})$ is assumed to be non-random. It can be assumed to depend on time, but the data analysis suggested that it is stable over time so we suppose no dependency on $t$ here. Furthermore, we factorize the residual field into

$$
\varepsilon(\mathbf{s} ; t)=\sigma(\mathbf{s} ; t) \epsilon(\mathbf{s} ; t)
$$

where $\sigma(\mathbf{s} ; t)$ is a nonrandom seasonal function, satisfying the condition

$$
\sigma(\mathbf{s} ; t)=\sigma(\mathbf{s} ; t+365)
$$

for any time $t$. Assume that

$$
\left\{\epsilon(\mathbf{s} ; t): \mathbf{s} \in D \subset R^{2}, t \in[0, \infty)\right\}
$$

is a zero-mean stationary Gaussian spatial-temporal random field which is independent in time and having a spatial correlation function defined by the parametric model

$$
\operatorname{corr}\left\{\epsilon(\mathbf{s} ; t), \epsilon\left(\mathbf{s}+\mathbf{h}_{\mathbf{s}} ; t\right)\right\}=\rho\left(\mathbf{h}_{\mathbf{s}} ; \theta_{\mathbf{s}}\right)
$$

for all $\mathbf{s}, \mathbf{s}+\mathbf{h}_{\mathbf{s}} \in D$. We assume that the cross-correlation in time and space is equal to zero, and note that the random field $\varepsilon(\mathbf{s} ; t)$ has the following properties:

A1. Residuals are uncorrelated but dependent in time.

A2. Residuals are correlated in space. 
The stated properties and assumptions lead us to the following model of the spatialtemporal covariance function for $\varepsilon(\mathbf{s} ; t)$ defined in $(3)$ :

$$
C\left(\mathbf{s}, \mathbf{s}+\mathbf{h}_{\mathbf{s}} ; \theta\right)=\rho\left(\mathbf{h}_{\mathbf{s}} ; \theta_{\mathbf{s}}\right) \Sigma_{\mathbf{s}}\left(\theta_{t}\right) \Sigma_{\mathbf{s}+\mathbf{h}_{\mathbf{s}}}\left(\theta_{t}\right),
$$

where $\Sigma_{\mathbf{s}}\left(\theta_{t}\right)$ is a diagonal variance matrix at the location $\mathbf{s} \in D$. We denote by $\theta=$ $\left(\theta_{\mathbf{s}} ; \theta_{t}\right)^{T} \in \Theta$ the $k \times 1$ parameter vector, where $\Theta$ is an open subset of $R^{k}$, in (6) and (7). Here $T$ means transposing.

First, we consider a temporal model appropriate for a temperature field at a single spatial location (measurement station), i.e. we deal with the time series of data from each station, independent on the spatial information. Denote by $Z_{i}(t)$ a time series at the spatial location $\mathbf{s}_{i} \in D, i=1, \ldots, n, t=1, \ldots, T$. Our spatial-temporal model considered at a single spatial location becomes then the time series

$$
Z_{i}(t)=\mu_{i}(t)+\varepsilon_{i}(t)
$$

where $\mu_{i}(t)$ and $\varepsilon_{i}(t)$ denote the mean and residual process at the moment $t=1, \ldots, T$ at the spatial location $\mathbf{s}_{i} \in D, i=1, \ldots, n$, respectively. Here

$$
\mu_{i}(t)=S_{i}(t)+\alpha_{i}\left(Z_{i}(t-1)-S_{i}(t-1)\right),
$$

where

$$
S_{i}(t)=a_{0}^{i}+a_{1}^{i} t+b_{1}^{i}+b_{2}^{i} \cos \left(2 \pi\left(t-b_{3}^{i}\right) / 365\right),
$$

describes the linear trend and the seasonality in DAT. The speed in which the DAT reverts back to its mean is denoted by $\alpha_{i}$.

We assume that the residual process $\varepsilon_{i}(t)$ is of the following form

$$
\varepsilon_{i}(t)=\sigma_{i}(t) \epsilon_{i}(t)
$$

where $\sigma_{i}(t)$ is a seasonally dependent standard deviation function, and $\epsilon_{i}(t)$ is a zero-mean temporally independent Gaussian random process with standard deviation equal to one. Here we assume that

$$
\sigma_{i}^{2}(t)=c_{1}^{i}+\sum_{k=1}^{4}\left[c_{2 k}^{i} \cos (2 k \pi t / 365)+c_{2 k+1}^{i} \sin ((2 k+1) \pi t / 365)\right] .
$$

In the subsection below we fit the suggested model to the Lithuanian DAT.

3.2. Model fitting. Alaton et al. (2002) and Campbell and Diebold (2005) consider data sets of DAT observed for more than 40 years in Sweden and the USA, respectively. They show that there has been a clear increase of DAT over the years. We check if there is a linear trend in Lithuanian DAT by running a simple linear regression. The obtained slopes are all approximately equal to 0.0001 as intercepts vary in the interval [5.46,6.91]. Values of these parameters for 16 stations are presented in Table 2 (columns 2 and 3). All obtained values are significant at the $1 \%$ level. Hence, we have a linear trend in DAT. This basically means that the DAT from the middle of 1964 to the middle of 2003 has increased approximately by $1.5^{\circ} \mathrm{C}$ in Lithuania. The positive trend corresponds to the increase in the global mean temperature, which has risen over the past hundred years by about $0.6^{\circ} C$ 
and is now increasing more rapidly. This phenomena is coursed by global warming. These facts have resulted in more extreme weather events such as droughts, heavy precipitation patterns, floods, hurricanes etc.

We eliminate the obtained linear trend from the DAT and model the seasonal effects of residuals. Already from Fig. 2 it is obvious that DAT follows certain periodical pattern which we describe with the function $S_{i}(t)$ in (9). Parameters of the seasonal part of this function are fitted using the nlinfit procedure in MATLAB, and the estimates are reported in Table 2 (columns 4,5 and 6 ).

Further we remove seasonal effects from DAT and apply an $A R(1)$-model, or meanreverting model for the obtained residuals. The values (see Table 2, last column) of the coefficients $\alpha_{i}$ are slightly above 0.80 for all 16 stations and do not vary much from station to station. All values are statistically significant at the $1 \%$ level. We also fitted the constant, which turned out to be statistically insignificant and therefore assumed to be equal to zero.

Autocorrelation functions of the residuals obtained after eliminating the linear and seasonal trends, and the influence of the mean reversion process, are presented in Fig. 5. The values of the autocorrelation function for the first two lags are quite high but decrease very rapidly and vary around zero for bigger lags. To get more information on the temporal dependencies in the residual process we analyze the autocorrelation function for the squares. Plots of the autocorrelation functions of squared residuals are given in Fig. 6 . Here we observe a clear seasonal pattern which is an indication of the time dependency in the variance of residuals, which we now model.

First, we calculate the daily empirical variance by averaging the values of the squared residuals of the particular day over all years. Then we model it by the truncated Fourier function (10). The fitted parameters of this function are presented in Table 3. In Fig. 7 we present the empirical $\sigma_{i}^{2}(t)$-functions together with fitted ones for each station separately. It is obvious that the fluctuations in cold season temperatures are considerably higher then those during warm season. This observation is consistent with the cold season's interchange of warm Atlantic air and cold Arctic air advection as well as atmosphere blocking activities, which produce strong extreme temperature anomalies and are stronger and more frequent during the cold season over north Atlantic-Europe sector (Stankūnavičius and Jalinskas, 2001).

Autocorrelation functions for residuals and squared residuals obtained after eliminating the seasonal dependency in the variance are presented in Fig. 8 and Fig. 9, respectively. The autocorrelation function of the residuals basically shows that we are left with zeromean uncorrelated noise. The autocorrelation function of squared residuals decays for the several first lags and then simply varies around zero, clearly indicating that we managed to remove the seasonality in the variance. The rapid decay of the correlation for the first few lags may be modelled using, for example, a GARCH-process (see Bollerslev (1986) for more on such processes and their properties).

In our model there are 15 parameters for each location. We want to have a spatial model for each of them in order to specify $Z(\mathbf{s} ; t)$. Note, that we are not interested in the intercept of the linear trend as it is the same for all stations, thus we deal with only 14 parameters. 

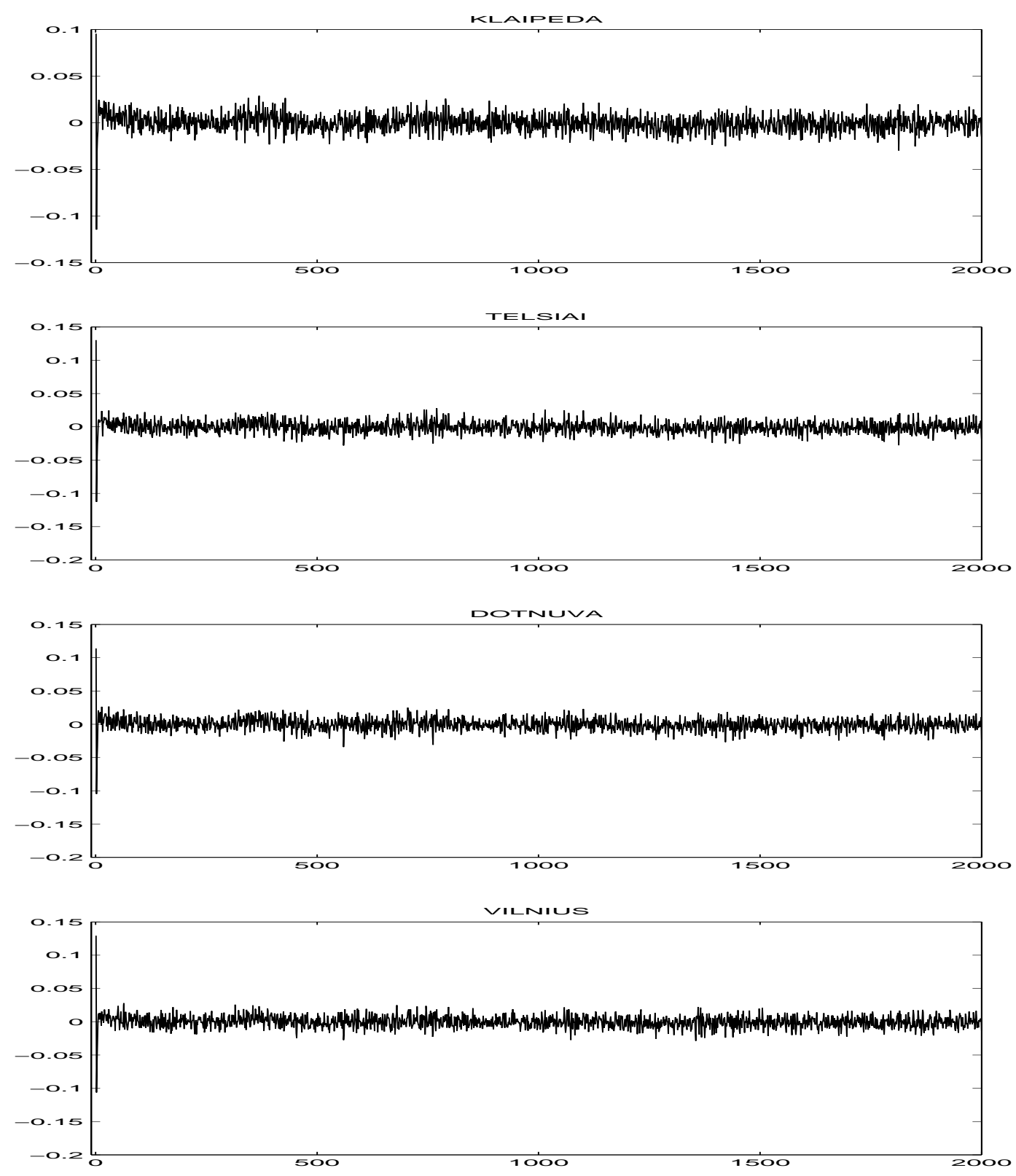

Figure 5. Autocorrelation functions of the residuals in Klaipèda, Telšiai, Dotnuva and Vilnius.

First we fit the plane

$$
P^{\delta}=\lambda_{0}^{\delta}+\lambda_{1}^{\delta} x+\lambda_{2}^{\delta} y
$$

to each set of parameters separately. Here $x$ and $y$ are $16 \times 1$ vectors of metric coordinates of stations, and $\delta$ indicates the set of parameters and takes the values $a_{1}, b_{j}, j=1,2,3, \alpha$ 

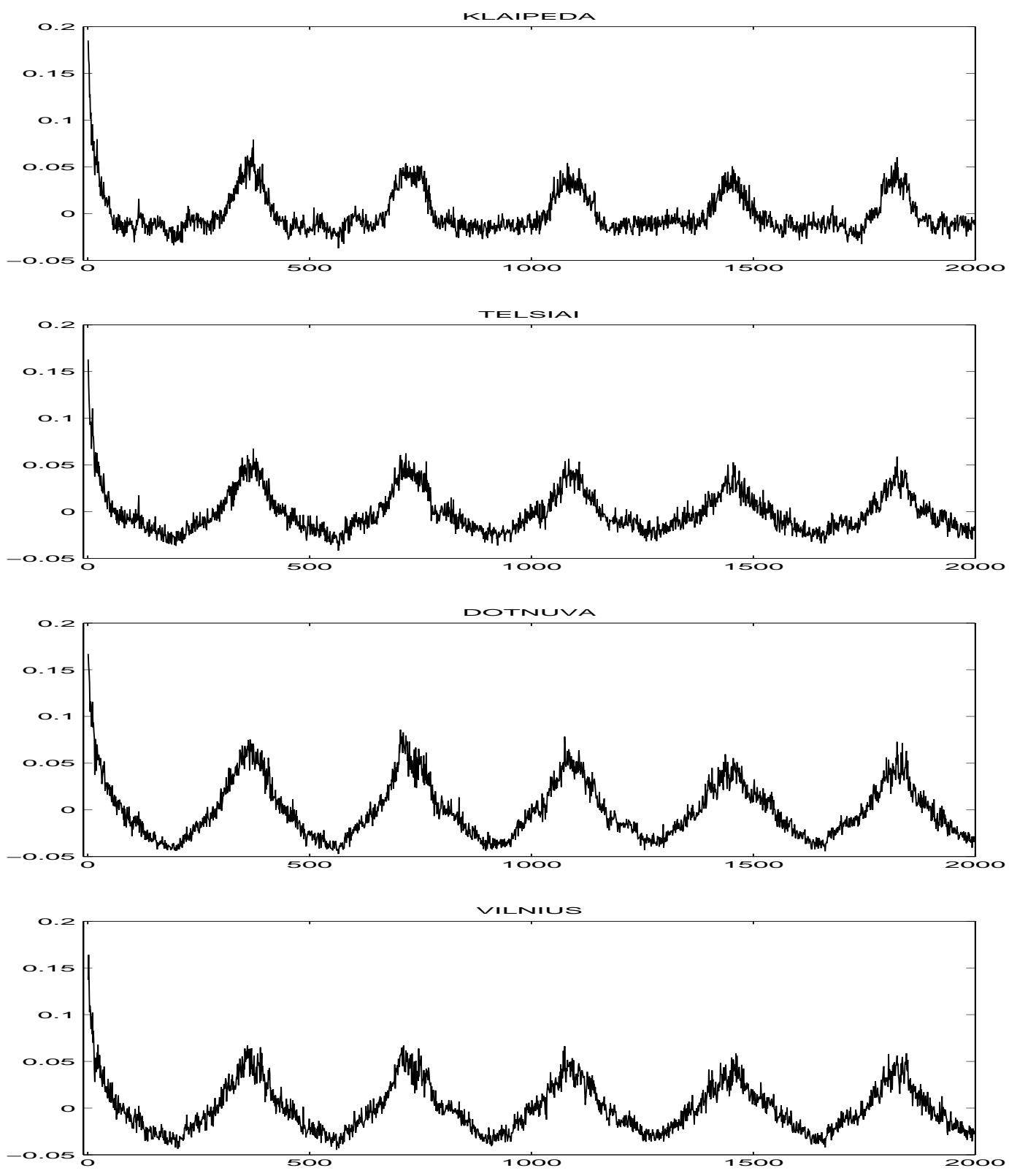

FiguRE 6. Autocorrelation functions of the squared residuals in Klaipedda, Telšiai, Dotnuva and Vilnius.

and $c_{k}, k=1, \ldots, 9$. The values of plane parameters and the corresponding mean square error $(M S E)$ values are given in Table 4 .

The histograms for residuals are close to normal (see Fig. 10). Similar indications we get when calculating the descriptive statistics characteristics analogous to those in Table 1. The 

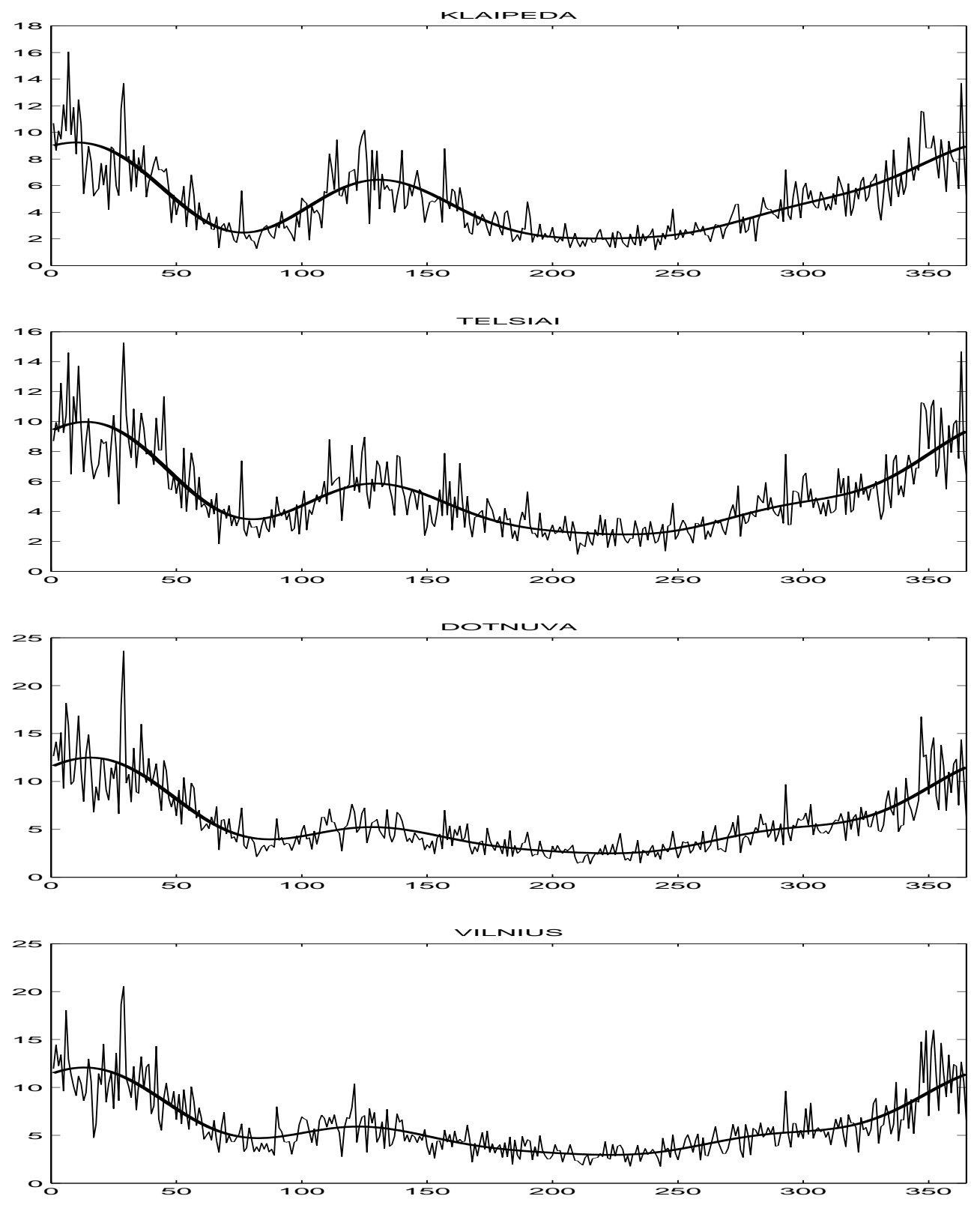

Figure 7. Empirical and fitted $\sigma_{i}^{2}(t)$ in Klaipèda, Telšiai, Dotnuva and Vilnius.

Kolmogorov-Smirnov test does not indicate clearly that the residuals are normal, however we think that our assumption about the Gaussian random field is quite reasonable.

Since the remaining residuals in each station are not temporally correlated, we can easily perform a spatial analysis based on them. We calculate the empirical correlations for each pair of stations giving us 120 values altogether. The plot of empirical correlations against 

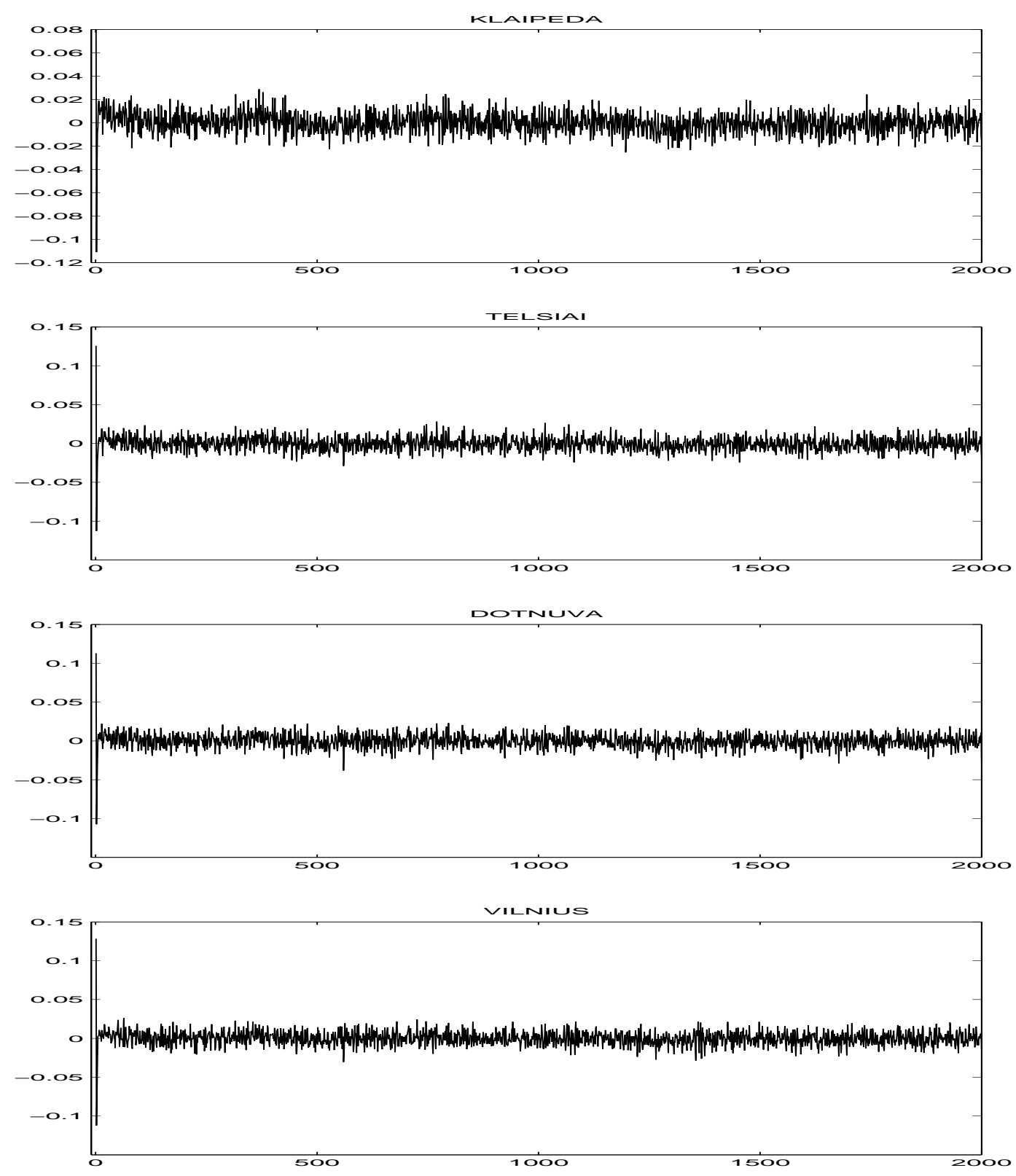

Figure 8. Autocorrelation function of the final residuals in Klaipeda, Telšiai, Dotnuva and Vilnius.

the corresponding distance between stations is given in Fig. 11. Distances were calculated in meters using the program ArcView GIS 3.2a. 

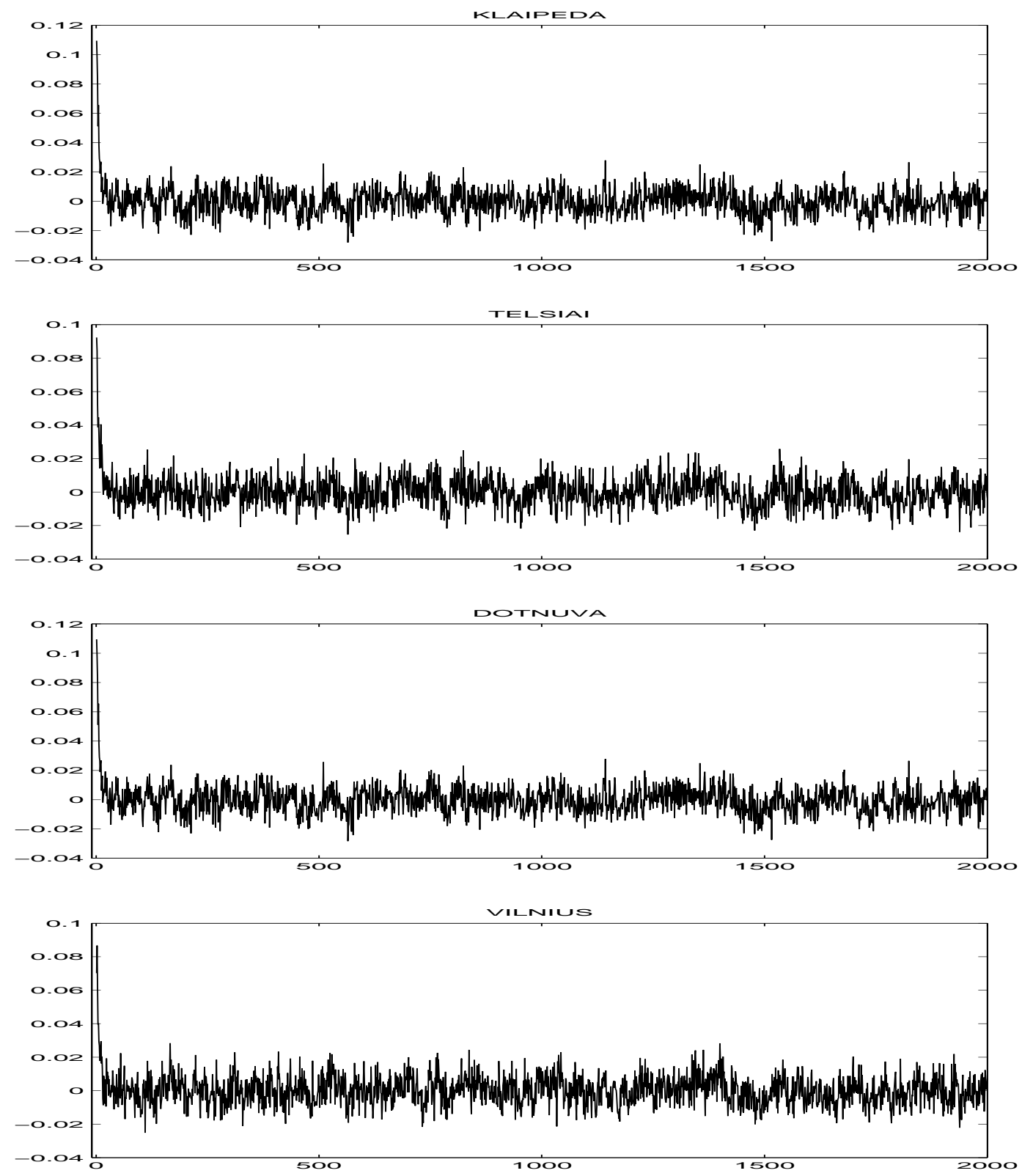

Figure 9. Autocorrelation function of the final squared residuals in Klaipėda, Telšiai, Dotnuva and Vilnius.

Using the nlinfit function of MATLAB we fitted the following spherical spatial correlation function to the empirical values

$$
\rho\left(\left|\mathbf{h}_{\mathbf{s}}\right| ; \theta_{\mathbf{s}}\right)=1-\frac{3}{2} \frac{\left|\mathbf{h}_{\mathbf{s}}\right|}{\theta_{\mathbf{s}}}+\frac{1}{2}\left(\frac{\left|\mathbf{h}_{\mathbf{s}}\right|}{\theta_{\mathbf{s}}}\right)^{3}
$$



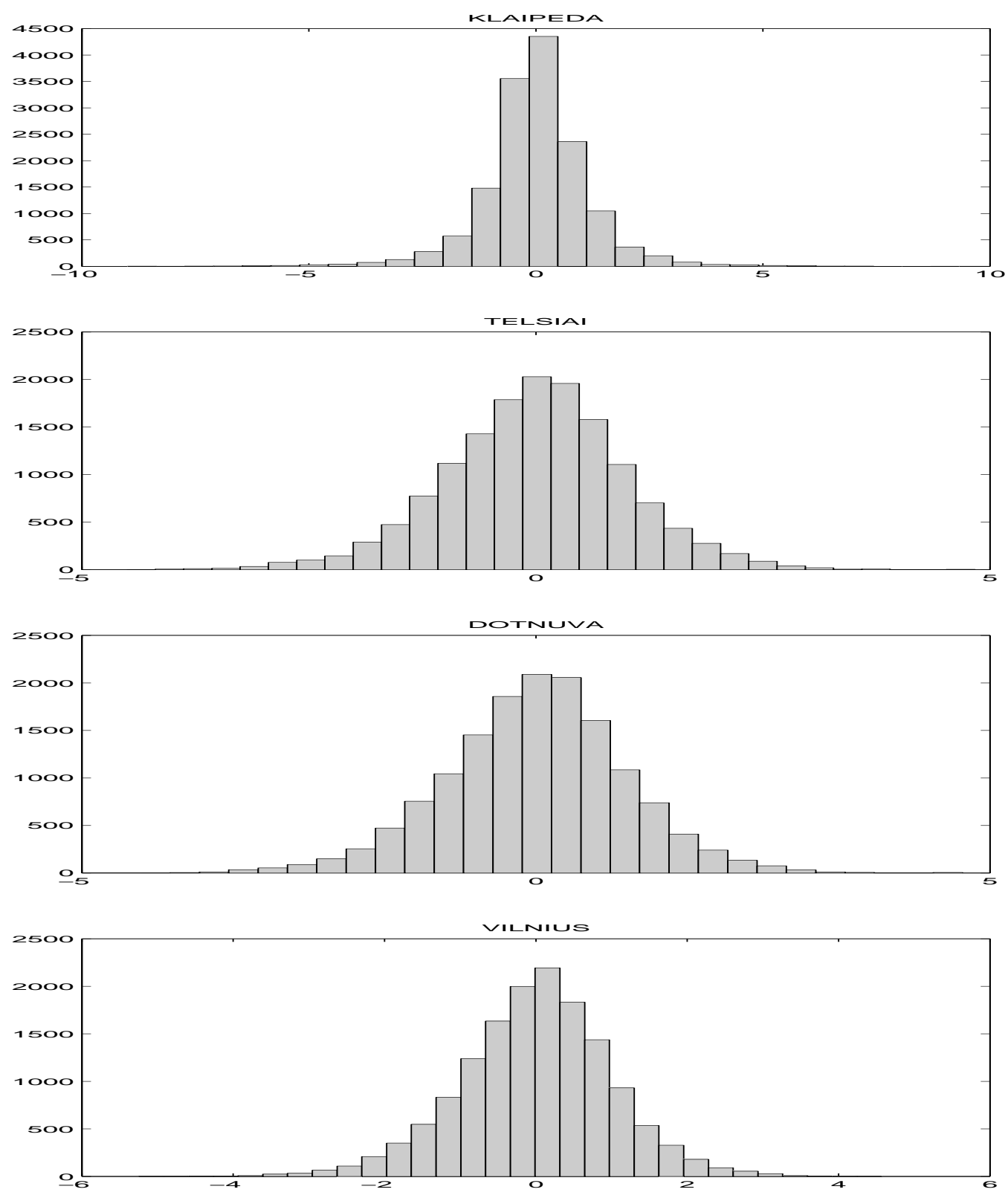

FiguRE 10. Histograms of the final residuals in Klaipèda, Telšiai, Dotnuva and Vilnius.

with $\theta_{\mathbf{s}}=1.1348 \times 10^{6}$. In Fig. 11 we also present the fitted spatial correlation function. In fact, we tried to fit several more functions to the empirical values of the correlations, however, none of them performed as good as the spherical correlation function. 


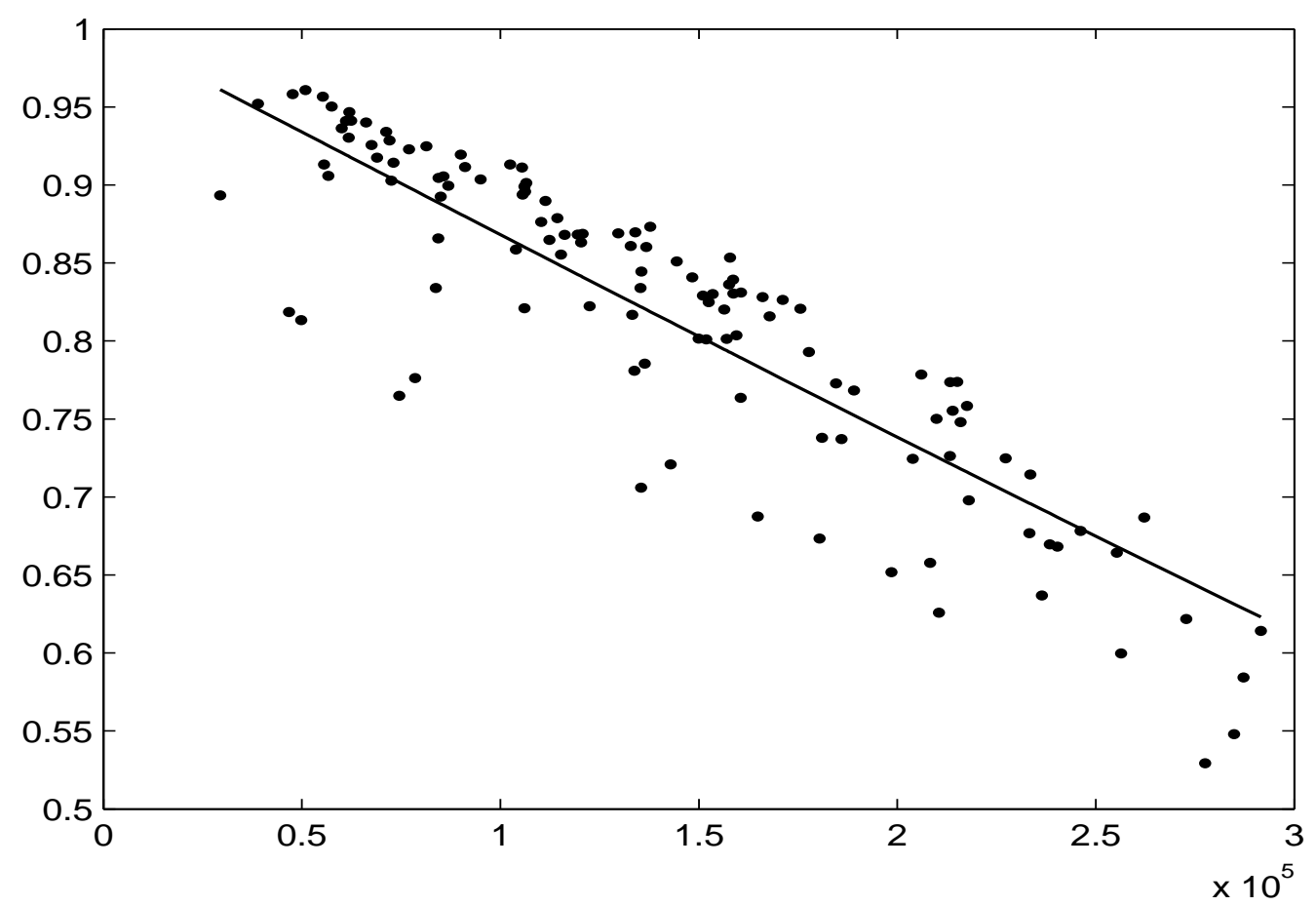

FiguRE 11. The empirical and fitted spatial correlation functions.

3.3. Model validation. It seems from the analysis above that the suggested spatialtemporal model fits Lithuanian DAT well. Here we validate our model in the following way.

There are 4 stations we did not use in our analysis. We use two of them, Palanga and Traku Voke, to validate our model. In Palanga we have a time series of data ranging from the 1st of June 1992 to the 31st of August 2004. This results in 4472 observations. In Traku Voke, the time series are much longer. Here we take the values of DAT from the 1st of June 1971 to the 31st of August 2004, which gives us 12146 observations. The choice of these two stations was not random, since Palanga is outside of the area made up by the 16 locations, but not far from other stations on the cost, and Trakụ Voke is within the area of the study and in addition it is just approximately $12 \mathrm{~km}$ away from the Vilnius station.

In Table 5 and Table 6 we present the values of the parameters estimated at the Palanga and Traku Voke stations, respectively, together with their standard errors, and the values of parameters obtained from our spatial model. It is clear that the model fits DAT quite well: most of the estimated values are within two standard errors from the fitted values.

We remark in passing that we tried to fit a quite general third-order trend surface model to the parameters as well. Naturally, values of $M S E$ where smaller in all cases. However, the differences in values of model parameters where only slightly different from the case of 
the plane. Taking into account the complexity of the third-order trend surface model we prefer the plane as a spatial model for the parameters of interest.

\section{Applications}

Here we will demonstrate how the suggested model can be applied to temperature prediction and classification. Before we proceed to the examples, let us make some additional assumptions.

We use the detrended and deseasonalized DAT in the examples below, i.e. we consider

$$
\bar{Z}_{i}(t)=\alpha_{i} X_{i}(t-1)+\varepsilon_{i}(t),
$$

where $X_{i}(t-1)=Z_{i}(t-1)-S_{i}(t-1)$. This simplifies the notation without affecting the analysis. We obtain the actual values for prediction by adding the trend and seasonal function $S_{i}(t)$. Such simplification does not affect the classification procedure at all.

Also, we make a simplifying assumption on the mean-reverting process parameter $\alpha_{i}$. We assume it to be a constant over space, i.e. $\alpha_{i}=\alpha$. This seems to be quite reasonable for Lithuania, as values of $\alpha_{i}$ do not vary much from station to station (see Table 2). Furthermore, we assume the variance to be the same for all locations, i.e. $\sigma_{i}^{2}(t)=\sigma^{2}(t)$, for $i=1, \ldots, n$.

The estimates for $\alpha$ and $\sigma^{2}(t)$ can be found in several different ways: by averaging the estimated parameters (see Table 2 and Table 3) over all 16 stations; by averaging the parameters obtained using the fitted planes (see Table 4); or by using maximum likelihood approach.

4.1. Prediction. One of the most interesting and important tasks in weather analysis is the prediction of future weather conditions at a specific location in space. Kriging is a well-known technique for spatial prediction (see e.g. Cressie (1993)), however, it is not difficult to generalize it to the spatial-temporal case. The generic problem is then to predict $Z\left(\mathbf{s}_{0} ; t_{0}\right), \mathbf{s}_{0} \in D$, from data $\left(Z\left(\mathbf{s}_{1} ; t\right), \ldots, Z\left(\mathbf{s}_{n} ; t\right)\right), t=1, \ldots, T$, where $t_{0}>T$ is a future time moment.

Here we apply the universal kriging approach to predict the DAT in Palanga station $\left(\mathbf{s}_{0}\right)$ for the time moment $t_{0}$, which we choose to be the 1st of September 2004 (it corresponds to $\left.t_{0}=4473\right)$, that is, we predict one day ahead based on our data sample, $t_{0}=T+1$.

Let us introduce the following notations: $\bar{U}(t)=\left(\bar{Z}_{1}(t), \ldots, \bar{Z}_{n}(t)\right)^{T}$, $X_{t-1}=\left(X_{1}(t-1), \ldots, X_{n}(t-1)\right)^{T}$, and denote by $R$ the $n \times n$ spatial correlation matrix with elements $\rho\left(\mathbf{h}_{\mathbf{s}} ; \theta_{\mathbf{s}}\right)$, and $C_{0}$ the $n \times 1$ vector consisting of the spatial correlations $\rho\left(\mathbf{s}_{i}-\mathbf{s}_{0} ; \theta_{\mathbf{s}}\right), i=1, \ldots, n$. Note that $n=16$ for our Lithuanian data set.

The universal kriging prediction is then given by the equation

$$
\widehat{\bar{Z}}_{0}\left(t_{0}\right)=a^{T} \bar{U}(T)
$$

where

$$
a=R^{-1} C_{0}+R^{-1} X_{T-1}\left(X_{T-1}^{T} R^{-1} X_{T-1}\right)^{-1}\left(X_{0}\left(t_{0}-1\right)-X_{T-1}^{T} R^{-1} C_{0}\right) .
$$


The corresponding mean square prediction error (mspe) is

$$
\begin{aligned}
\operatorname{mspe}\left(\widehat{\bar{Z}}_{0}\left(t_{0}\right)\right)=\sigma^{2}\left(t_{0}\right)- & C_{0}^{T} R^{-1} C_{0}+\left(X_{0}(T)-X_{T-1}^{T} R^{-1} C_{0}\right)^{2} \times \\
& \left(X_{T-1}^{T} R^{-1} X_{T-1}\right)^{-1} .
\end{aligned}
$$

As we do not know the true value of $\sigma^{2}\left(t_{0}\right)$, we estimate it using three different methods and substitute the unknown value by one of the estimators.

Method $A$. We average the estimated parameters of $\sigma_{i}^{2}(t)$ from Table 3 over 16 stations. Then $\hat{\sigma}_{A}^{2}\left(t_{0}\right)=4.134$.

Method $B$. We average the parameters of $\sigma_{i}^{2}(t)$ obtained by fitted planes (Table 4$)$. In this case $\hat{\sigma}_{B}^{2}\left(t_{0}\right)=4.131$.

Note that Method $A$ and Method $B$ can be used for the estimation of the parameter $\alpha$ as well.

Method $C$. We use the maximum likelihood estimator of $\sigma^{2}(t)$ :

$$
\widehat{\sigma}_{C}^{2}(t)=\frac{1}{n-1}(\bar{U}(t)-\widehat{\bar{\mu}}(t))^{T} R^{-1}(\bar{U}(t)-\widehat{\bar{\mu}}(t)),
$$

where $\widehat{\bar{\mu}}(t)=\widehat{\alpha} X_{t-1}$ is a maximum likelihood estimator of $\bar{\mu}(t)$ with

$$
\widehat{\alpha}=\left(X_{t-1}^{T} R^{-1} X_{t-1}\right)^{-1} X_{t-1}^{T} R^{-1} \bar{U}(t) .
$$

Then $\hat{\sigma}_{C}^{2}\left(t_{0}\right)=4.224$.

After performing simple calculations we get that $\widehat{\bar{Z}}_{0}\left(t_{0}\right)=8.3$ with $\operatorname{mspe}_{A}\left(\widehat{\bar{Z}}_{0}\left(t_{0}\right)\right)=$ $3.18, \operatorname{mspe}_{B}\left(\widehat{\bar{Z}}_{0}\left(t_{0}\right)\right)=3.17$, and $\operatorname{mspe}_{C}\left(\widehat{\bar{Z}}_{0}\left(t_{0}\right)\right)=3.55$, here index $A, B$ or $C$ indicates the type of the estimator of $\sigma^{2}\left(t_{0}\right)$.

Taking into consideration the values of the linear and seasonal trend at $t_{0}=4473$, i.e. the 1st of September 2004, we get the predicted DAT $\widehat{Z}_{0}\left(t_{0}\right)=16.1^{0} \mathrm{C}$. Just for the comparison we mention that the observed DAT one day earlier (i.e. the 31st of August 2004) in Palanga was $16.8^{\circ} \mathrm{C}$, as two days earlier it was $18.9^{\circ} \mathrm{C}$, and even more, the true value of DAT in Palanga on the day when prediction was made was $15.1^{\circ} \mathrm{C}$. We have reasons to believe that our model predicts the DAT well. The values of mspe are not large indicating that the prediction is quite reliable. They are also very similar for all three types of estimators, giving a clear indication that the suggested model captures well the different phenomena in DAT.

It is often of interest to predict the weather for more days ahead. In order to do so we can iterate our temperature model to get

$$
Z_{i}(t+k)=S_{i}(t+k)+\alpha^{k}\left(Z_{i}(t)-S_{i}(t)\right)+\sum_{j=1}^{k} \alpha^{j} \varepsilon(t+j)
$$

for $k \geq 1$. We observe that this is again a linear Gaussian model which has the same structure as the case $k=1$ considered above. We do not go into further details about the prediction for $k$ bigger than one. 
4.2. Classification. There are several reasons of the different mesoclimate conditions in Lithuania, which are considered in climate classification schemes: advection of sea air, its primary transformation and sea breeze at the coastal part of Lithuania; orographic air-mass uplift accompanied with activation of frontal areas on the windward slopes of the height terrains; adiabatic descent on the downwind slopes of the height terrains; air turbulence and intensification of the thermal convection in the hilly (as well as laky and forested) areas; forested areas with increased radiation balance; hydrological and hydrogeological soil characteristics (Bukantis, 1994). Kaušyla's (1982) Lithuanian climatic regionalism scheme, based on the genetic scheme of climate classification made by Alisov (1954) is used in this study (see Fig. 1). From this point of view the territory of Lithuania is divided into the following regions: Seacoast (I), Žemaitija (Samogitia) (II), Central lowland (III), and Southeast highland (IV), which further are divided into subregions (not shown).

These regions are defined on the basis of many parameters like air temperature, wind parameters, air humidity values, atmospheric pressure, precipitation and their intensity, sunshine duration, meteorological parameters of the soil, etc. One may be interested if temperature alone could be a sufficient variable for describing the climate regions. To answer such a question we suggest to run a classification procedure. In this subsection we illustrate how discriminant analysis could be applied for DAT in the case of two classes.

We assume that the first class $\Omega_{1}$ is Seacoast, consisting of the stations Nida, Klaipèda and Šilute. The second one $\Omega_{2}$ consists of the 13 remaining stations where all the three inland regions are merged into one. In this set-up, the number of stations in $\Omega_{1}$ and $\Omega_{2}$ is $n_{1}=3$ and $n_{2}=13$, respectively. We choose Palanga as the station to be classified for the time moment $t_{0}=4473$ corresponding to the 1st of September 2004. Remark in passing that as we can see from the Fig. 1, Palanga belongs to the class (or climate region) $\Omega_{1}$. Thus, we expect that the classification rule will assign Palanga station to the class $\Omega_{1}$. But at the same time we observe that the station is close to the border of the inland regions.

Here we use the plug-in Bayes classification rule (BCR), and calculate the actual error rate $(A E R)$. More detailed description of the procedure can be found in, for example, Šaltytè-Benth and Dučinskas (2005). Now we present briefly the necessary mathematics taken from that paper and adopted to the present model. Note that all the required conditions there hold for our model.

Denote by $\bar{Z}_{0}\left(t_{0}\right)$ the observation in Palanga at the time moment $t_{0}=T+1$, and let $\bar{z}_{0}\left(t_{0}\right)$ be a realization of it. Define $\pi_{l}$ as a prior probability and

$$
p_{l}\left(\bar{z}_{0}\left(t_{0}\right) ; \Psi\right)=\left(2 \pi \sigma^{2}\left(t_{0}\right)\right)^{-\frac{1}{2}} \exp \left(-\frac{1}{2 \sigma^{2}\left(t_{0}\right)}\left(\bar{z}_{0}\left(t_{0}\right)-\bar{\mu}_{l}^{0}\left(t_{0}\right)\right)^{2}\right)
$$

as the probability density function of $\bar{Z}_{0}\left(t_{0}\right)$ in $\Omega_{l}, l=1,2$. Here, $\Psi=\left(\sigma^{2}\left(t_{0}\right), \bar{\mu}_{1}^{0}\left(t_{0}\right), \bar{\mu}_{2}^{0}\left(t_{0}\right)\right)$ is a vector of (usually unknown) parameters, the variance $\sigma^{2}\left(t_{0}\right)$, which is assumed to be the same for all locations in both classes, and means $\bar{\mu}_{l}^{0}\left(t_{0}\right)=\alpha X_{0}(T), l=1,2$. The variance $\sigma^{2}\left(t_{0}\right)$ and the parameter of the mean-revertion model $\alpha$ can be estimated using Method $A, B$, or $C$. When using $\operatorname{Method} A$ or $B$, we calculate the estimators of $\alpha$ and $\sigma^{2}\left(t_{0}\right)$ separately for each class, and take a weighted average of the two $\sigma^{2}\left(t_{0}\right)$ estimators with weights proportional to the number of stations in the corresponding class. Note also 
that the maximum likelihood estimators (11) and (12) in the case of two classes are

$$
\widehat{\sigma}^{2}(t)=\frac{1}{n-1} \sum_{l=1}^{2}\left(\bar{U}_{l}(t)-\widehat{\bar{\mu}}_{l}(t)\right)^{T} R_{l}^{-1}\left(\bar{U}_{l}(t)-\widehat{\bar{\mu}}_{l}(t)\right),
$$

where $\widehat{\bar{\mu}}_{l}(t)=\widehat{\alpha}_{l} X_{l, t-1}$ is a maximum likelihood estimator of $\bar{\mu}_{l}(t)$ with

$$
\widehat{\alpha}_{l}=\left(X_{l, t-1}^{T} R_{l}^{-1} X_{l, t-1}\right)^{-1} X_{l, t-1}^{T} R_{l}^{-1} \bar{U}_{l}(t) .
$$

Here $\bar{U}_{l}(t)=\left(\bar{Z}_{l, 1}(t), \ldots, \bar{Z}_{l, n_{l}}(t)\right)^{T}$ is a so-called training sample from the class $\Omega_{l}, X_{l, t-1}=$ $\left(X_{l, 1}(t-1), \ldots, X_{l, n_{l}}(t-1)\right)^{T}$, and $R_{l}$ is a $n_{l} \times n_{l}$ spatial correlation matrix with elements $\rho\left(\mathbf{h}_{\mathbf{s}} ; \theta_{\mathbf{s}}\right), l=1,2$. Note that the estimators (13) and (14) are both unbiased, and $n=$ $n_{1}+n_{2}$.

Further we assume that $\bar{Z}_{0}\left(t_{0}\right)$ is independent on the training sample.

The plug-in BCR $d_{B}\left(\bar{z}_{0}\left(t_{0}\right) ; \widehat{\Psi}\right)=\arg \max _{\{l=1,2\}} \pi_{l} p_{l}\left(\bar{z}_{0}\left(t_{0}\right) ; \widehat{\Psi}\right)$ is obtained when the unknown parameters are replaced with their estimators in the BCR; here

$$
\widehat{\Psi}=\left(\widehat{\bar{\mu}}_{1}^{0}\left(t_{0}\right), \widehat{\bar{\mu}}_{2}^{0}\left(t_{0}\right), \widehat{\sigma}^{2}\left(t_{0}\right)\right) .
$$

We note that under the assumption that the variances in both classes are the same, the sample discriminant function corresponding to the plug-in BCR is a linear one and is defined as

$$
L\left(\bar{z}_{0}\left(t_{0}\right) ; \widehat{\Psi}\right)=\frac{1}{\widehat{\sigma}^{2}\left(t_{0}\right)}\left(\bar{z}_{0}\left(t_{0}\right)-\frac{\widehat{\bar{\mu}}_{1}^{0}\left(t_{0}\right)+\widehat{\widehat{\mu}}_{2}^{0}\left(t_{0}\right)}{2}\right)\left(\widehat{\bar{\mu}}_{1}^{0}\left(t_{0}\right)-\widehat{\bar{\mu}}_{2}^{0}\left(t_{0}\right)\right)+\gamma,
$$

where $\gamma=\ln \frac{\pi_{1}}{\pi_{2}}$.

Usually the prior probabilities $\pi_{l}, l=1,2$, are not known either. We assume that the classes are not changing with the time. Then we can let the prior probabilities be simply a proportion of the number of stations in each of the classes, which results in the following values: $\widehat{\pi}_{1}=0.19$ and $\widehat{\pi}_{2}=0.81$.

The $A E R$ for $d_{B}\left(\bar{z}_{0}\left(t_{0}\right) ; \widehat{\Psi}\right)$ in the considered case can be written as

$$
P\left(t_{0} ; \widehat{\Psi}\right)=\sum_{l=1}^{2} \pi_{l} \Phi\left((-1)^{l} \frac{\left(\bar{\mu}_{l}^{0}\left(t_{0}\right)-\frac{\widehat{\bar{\mu}}_{1}^{0}\left(t_{0}\right)+\widehat{\bar{\mu}}_{2}^{0}\left(t_{0}\right)}{2}\right) \frac{\widehat{\bar{\mu}}_{1}^{0}\left(t_{0}\right)-\widehat{\bar{\mu}}_{2}^{0}\left(t_{0}\right)}{\widehat{\sigma}^{2}\left(t_{0}\right)}+\gamma}{\sqrt{\frac{\left(\widehat{\bar{\mu}}_{1}^{0}\left(t_{0}\right)-\widehat{\bar{\mu}}_{2}^{0}\left(t_{0}\right)\right)^{2}}{\widehat{\sigma}^{4}\left(t_{0}\right)} \sigma^{2}\left(t_{0}\right)}}\right),
$$

where $\Phi$ is the standard normal distribution function. Here, $\pi_{l}$ will be substituted with $\widehat{\pi}_{l}$. Since we do not know the true values of the means and the variance, we use their estimators obtained by Method $A$ and $B$, and their maximum likelihood estimators from (13) and $(14)$ in $P\left(t_{0} ; \widehat{\Psi}\right)$.

The values of $\alpha_{l}$ and $\sigma^{2}(t)$ obtained using Method $A$ and Method $B$ were very similar for two classes. These values were slightly more different in the case when the maximum likelihood estimators (Method $C$ ) were used. This gives a very small value of the Machalanobis 
distance

$$
\Delta=\sqrt{\frac{\left(\widehat{\bar{\mu}}_{1}^{0}\left(t_{0}\right)-\widehat{\bar{\mu}}_{2}^{0}\left(t_{0}\right)\right)^{2}}{\widehat{\sigma}^{2}\left(t_{0}\right)}}
$$

(which also is a denominator in the function $\Phi$ in $A E R$ ). Small values of $\Delta$ indicate that there is no clear difference between two classes.

For all three estimation methods the value of the plug-in BCR is equal to 2, which means that Palanga station should be classified as coming from the class $\Omega_{2}$. The value of $A E R$ for all three cases of estimators is approximately 0.19. First, the $A E R$ is quite high, indicating that the discriminant analysis performed not very well. This also supports the conclusion for the small value of $\Delta$. On the other hand, the fact that Palanga is assigned to the class $\Omega_{2}$ is a clear sign that temperature alone is not a good indicator of these regions, perhaps as expected since there are many factors making up them. Also, it is likely that merging three inland climate regions to the one for this example was not reasonable.

Remark that climate regions are built on the basis of the long term data. Thus, the maximum likelihood estimators are not appropriate in the considered context, even if the results were very similar to those obtained when using Method $A$ and $B$.

As we show, it is simple to apply the discriminant analysis procedure in the case of two classes, and we believe that for a larger geographical region with bigger changes in the temperature it would perform much better. However, we also show that with the definition of the two classes used, temperature is not a good indicator to associate membership to a region.

Discriminant analysis could be applied in prediction as well. For example, rather than using data from all stations in Lithuania, one could use only data from those which belong to the same class as the station in question. Such an alternative approach based on discriminant analysis could be especially useful in circumstances where the number of stations is large.

\section{REFERENCES}

[1] Alaton P., Djehiche B., Stillberger D. (2002). On modeling and pricing weather derivatives. Appl. Math. Finance, $\mathbf{9}(1), 1-20$.

[2] Alisov B.P. (1954). Die Klimate der Erde. Deutscher Verlag der Wissenschaften, Berlin.

[3] Benth F.E., Šaltytè-Benth J. (2005). Stochastic modelling of temperature variations with a view towards weather derivatives. Appl. Math. Finance, 12(1), 53-85.

[4] Benth F.E., Šaltytė-Benth J. (2005). The volatility of temperature and pricing of weather derivatives. E-print, University of Oslo.

[5] Bollerslev T. (1986). Generalized autoregressive conditional heteroscedasticity. J. Econometrics, 31, 307-327.

[6] Brody D.C., Syroka J., Zervos M. (2002). Dynamical pricing of weather derivatives. Quantitative Finance, 3, 189-198.

[7] Bukantis A. (1994). Climate of Lithuania. Vilnius University Press (in Lithuanian).

[8] Campbell S.D., Diebold F.X. (2005). Weather forecasting for weather derivatives. J. Amer. Statist. Assoc., 100(469), 6-16. 
[9] Handcock M.S., Wallis J.R. (1994). An approach to statistical spatial-temporal modeling of meteorological fields. J. Amer. Statist. Assoc., 89(426), 368-390.

[10] Kaušyla K. (1982). Agro climate potential of Lithuania SSR and its estimation methods. Regional meteorology, 11, 35-46 (in Lithuanian).

[11] Rasmusson E.M., Dickinson R.E., Kutzback J.E., Cleveland M.K. (1993). Climatology. In "Handbook of Hydrology", Maidment D.R. (editor), McGraw-Hill Professional.

[12] Stankūnavičius G., Jalinskas P. (2001). Blocking activities in Europe and weather anomalies in Lithuania. Geographical Yearbook, XXXIV(1), 7-21.

[13] Šaltytė-Benth J., Dučinskas K. (2005). Linear discriminant analysis of multivariate spatial-temporal regressions. Scand. J. Statist., 32, 281-294. 
TABLE 1. Descriptive statistics characteristics

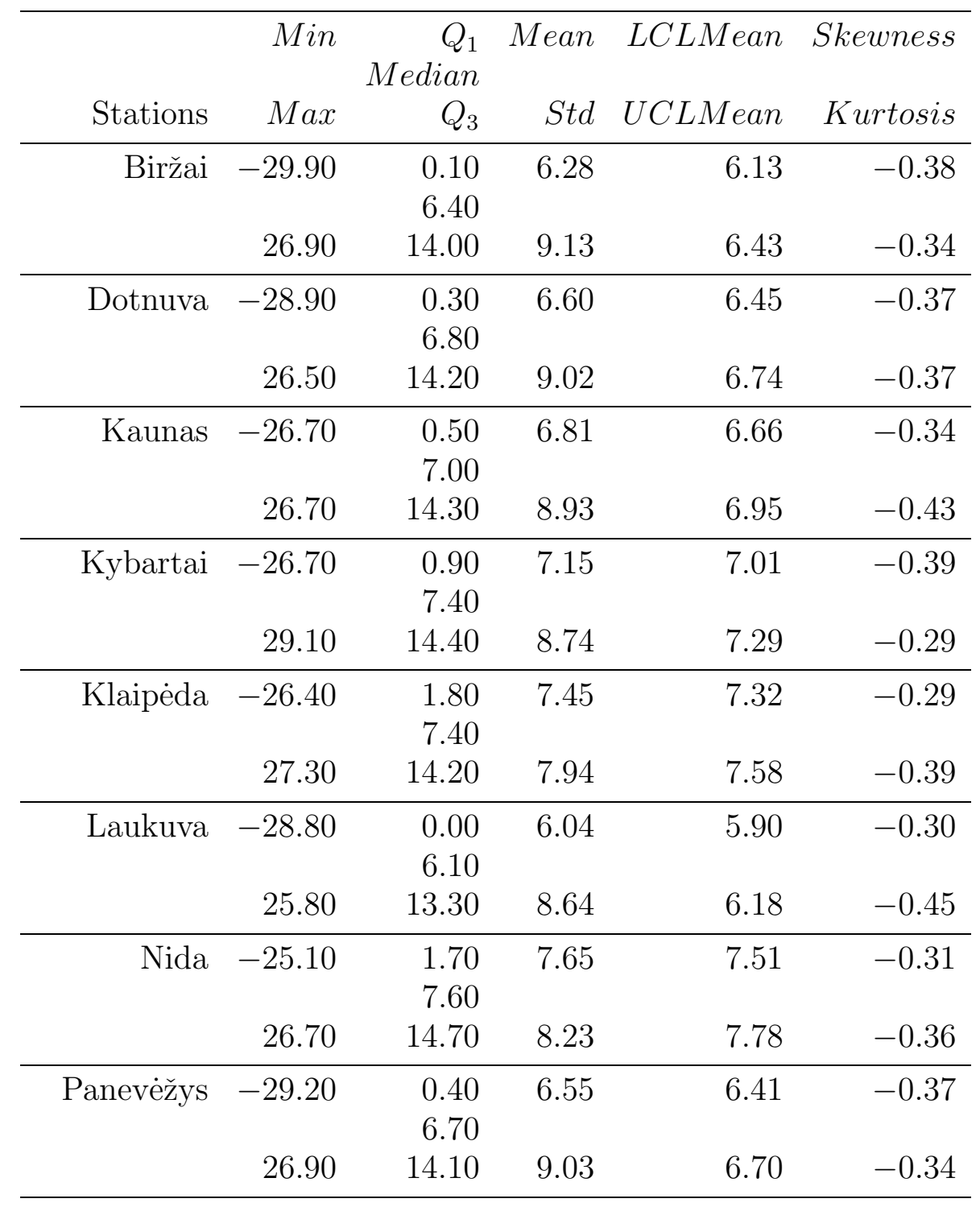

(Fred Espen Benth), Centre of Mathematics for Applications, Department of Mathematics, University of Oslo, P.O. Box 1053, Blindern, N-0316 Oslo, Norway, And, Agder University College, Department of Economics and Business Administration, Serviceboks 422, N-4604 Kristiansand, Norway

E-mail address: fredb@math.uio.no

URL: http://www.math.uio.no/ ${ }^{\sim}$ redb/

(Jūratė Šaltytė-Benth), Centre of Mathematics for Applications, Department of Mathematics, University of Oslo, P.O. Box 1053, Blindern, N-0316 Oslo, Norway, and, Department of System Research, Klaipéda University, H. Manto 84, LT-5808 Klaipėda, Lithuania

E-mail address: jurate@math.uio.no

(Paulius Jalinskas), Vilnius University, M.K. Ciurlionio 21/27, LT-03101 Vilnius, Lithuania, and, Lithuanian Hydrometeorological Service, Rudnios 6, LT-03900, Vilnius, Lithuania

E-mail address: paulius@meteo.lt 


\begin{tabular}{|c|c|c|c|c|c|}
\hline \multirow[t]{2}{*}{ Raseiniai } & -28.20 & $\begin{array}{l}0.20 \\
6.40\end{array}$ & 6.30 & 6.15 & -0.34 \\
\hline & 26.50 & 13.70 & 8.81 & 6.44 & -0.40 \\
\hline \multirow[t]{3}{*}{ Šiauliai } & -29.70 & 0.30 & 6.46 & 6.32 & -0.34 \\
\hline & & 6.50 & & & \\
\hline & 27.30 & 14.00 & 8.91 & 6.60 & -0.36 \\
\hline \multirow[t]{3}{*}{ Šilutė } & -26.10 & 1.20 & 7.15 & 7.01 & -0.34 \\
\hline & & 7.20 & & & \\
\hline & 26.80 & 14.20 & 8.39 & 7.29 & -0.31 \\
\hline \multirow[t]{3}{*}{ Telšiai } & -29.60 & 0.30 & 6.34 & 6.20 & -0.28 \\
\hline & & 6.40 & & & \\
\hline & 27.40 & 13.50 & 8.58 & 6.48 & -0.45 \\
\hline \multirow[t]{3}{*}{ Ukmergè } & -28.40 & 0.30 & 6.60 & 6.46 & -0.39 \\
\hline & & 6.80 & & & \\
\hline & 28.20 & 14.20 & 9.12 & 6.75 & -0.29 \\
\hline \multirow[t]{3}{*}{ Utena } & -29.20 & 0.00 & 6.22 & 6.07 & -0.39 \\
\hline & & 6.40 & & & \\
\hline & 27.70 & 14.00 & 9.18 & 6.37 & -0.33 \\
\hline \multirow[t]{3}{*}{ Varèna } & -27.70 & 0.30 & 6.49 & 6.35 & -0.41 \\
\hline & & 6.70 & & & \\
\hline & 28.00 & 14.10 & 9.12 & 6.64 & -0.24 \\
\hline \multirow[t]{2}{*}{ Vilnius } & -28.30 & $\begin{array}{r}-0.40 \\
6.40\end{array}$ & 6.15 & 6.00 & -0.32 \\
\hline & 27.40 & 14.00 & 9.28 & 6.30 & -0.51 \\
\hline
\end{tabular}


TABLE 2. Fitted parameters of the linear trend, seasonal trend and mean-revertion

\begin{tabular}{rrrrrrr}
\hline Stations & $a_{0}^{i}$ & $a_{1}^{i}$ & $b_{1}^{i}$ & $b_{2}^{i}$ & $b_{3}^{i}$ & $\alpha_{i}$ \\
\hline Biržai & 0.0001 & 5.51 & -0.02 & 11.38 & 44.51 & 0.82 \\
Dotnuva & 0.0001 & 5.79 & 0.01 & 11.24 & 44.73 & 0.82 \\
Kaunas & 0.0001 & 6.24 & -0.23 & 11.16 & 44.59 & 0.82 \\
Kybartai & 0.0001 & 6.45 & -0.09 & 10.80 & 45.38 & 0.82 \\
Klaipėda & 0.0001 & 6.77 & -0.11 & 9.87 & 52.49 & 0.81 \\
Laukuva & 0.0001 & 5.46 & -0.21 & 10.76 & 46.01 & 0.83 \\
Nida & 0.0001 & 6.91 & -0.05 & 10.39 & 52.32 & 0.83 \\
Panevėžys & 0.0001 & 5.79 & -0.03 & 11.23 & 44.49 & 0.82 \\
Raseiniai & 0.0001 & 5.69 & -0.19 & 10.98 & 45.18 & 0.83 \\
Šiauliai & 0.0001 & 5.78 & -0.11 & 11.08 & 45.80 & 0.82 \\
Šilutė & 0.0001 & 6.49 & -0.13 & 10.39 & 47.03 & 0.82 \\
Telšiai & 0.0001 & 5.57 & -0.02 & 10.68 & 46.52 & 0.83 \\
Ukmergè & 0.0001 & 5.90 & -0.09 & 11.30 & 44.46 & 0.81 \\
Utena & 0.0001 & 5.52 & -0.09 & 11.38 & 44.22 & 0.81 \\
Varėna & 0.0001 & 5.91 & -0.21 & 11.25 & 44.16 & 0.80 \\
Vilnius & 0.0001 & 5.51 & -0.15 & 11.61 & 43.70 & 0.82 \\
\hline
\end{tabular}

TABLE 3. Fitted parameters of $\sigma_{i}^{2}(t)$ function

\begin{tabular}{rrrrrrrrrr}
\hline Stations & $c_{1}^{i}$ & $c_{2}^{i}$ & $c_{3}^{i}$ & $c_{4}^{i}$ & $c_{5}^{i}$ & $c_{6}^{i}$ & $c_{7}^{i}$ & $c_{8}^{i}$ & $c_{9}^{i}$ \\
\hline Biržai & 6.00 & 3.85 & 1.05 & 1.69 & 0.29 & 0.86 & 1.28 & -0.09 & 0.53 \\
Dotnuva & 5.69 & 3.40 & 0.91 & 1.56 & 0.12 & 0.81 & 1.09 & 0.04 & 0.57 \\
Kaunas & 5.56 & 2.92 & 0.85 & 1.34 & 0.02 & 0.83 & 0.92 & 0.10 & 0.55 \\
Kybartai & 5.80 & 2.79 & 0.92 & 1.27 & 0.03 & 0.94 & 0.93 & 0.08 & 0.55 \\
Klaipėda & 4.72 & 2.05 & 0.74 & 1.30 & -1.05 & 1.06 & 0.80 & -0.17 & 0.51 \\
Laukuva & 5.21 & 2.47 & 0.89 & 1.34 & -0.27 & 0.86 & 0.87 & -0.02 & 0.50 \\
Nida & 4.11 & 2.24 & 1.10 & 1.79 & -0.12 & 0.78 & 1.05 & -0.16 & 0.36 \\
Panevěžys & 5.94 & 3.56 & 0.91 & 1.55 & 0.15 & 0.88 & 1.13 & 0.03 & 0.52 \\
Raseiniai & 5.45 & 2.85 & 0.82 & 1.50 & -0.02 & 0.86 & 1.01 & 0.05 & 0.52 \\
Šiauliai & 5.67 & 3.01 & 1.07 & 1.48 & 0.02 & 0.82 & 1.11 & -0.07 & 0.50 \\
$\check{\text { Šilutė }}$ & 5.31 & 2.48 & 0.82 & 1.34 & -0.48 & 1.05 & 0.96 & 0.02 & 0.53 \\
Telšiai & 5.05 & 2.26 & 0.90 & 1.26 & -0.41 & 0.85 & 0.84 & -0.05 & 0.52 \\
Ukmergė & 6.43 & 3.45 & 1.23 & 1.42 & 0.35 & 0.95 & 1.28 & 0.03 & 0.57 \\
Utena & 6.51 & 3.89 & 1.21 & 1.54 & 0.32 & 0.97 & 1.22 & 0.06 & 0.52 \\
Varėna & 7.01 & 3.98 & 0.90 & 1.31 & 0.41 & 1.05 & 1.15 & 0.23 & 0.53 \\
Vilnius & 5.95 & 3.03 & 0.94 & 1.33 & -0.04 & 0.92 & 0.86 & 0.17 & 0.54 \\
\hline
\end{tabular}


TABLE 4. Fitted parameters of the plane and corresponding mean square error $(M S E)$

\begin{tabular}{rrrrr}
\hline$\delta$ & $\lambda_{0}^{\delta}$ & $\lambda_{1}^{\delta}$ & $\lambda_{2}^{\delta}$ & $M S E$ \\
\hline$a_{1}$ & 29.91 & $-36.61 \times 10^{-7}$ & $-36.27 \times 10^{-7}$ & 0.087 \\
$b_{1}$ & -4.59 & $83.46 \times 10^{-9}$ & $72.34 \times 10^{-8}$ & 0.004 \\
$b_{2}$ & -2.00 & $41.38 \times 10^{-7}$ & $18.02 \times 10^{-7}$ & 0.063 \\
$b_{3}$ & 127.59 & $-19.69 \times 10^{-6}$ & $-11.82 \times 10^{-6}$ & 3.561 \\
$\alpha$ & 0.51 & $-32.85 \times 10^{-9}$ & $52.52 \times 10^{-9}$ & $3.19 \times 10^{-5}$ \\
$c_{1}$ & 3.17 & $56.06 \times 10^{-7}$ & $-16.97 \times 10^{-9}$ & 0.208 \\
$c_{2}$ & -11.21 & $55.75 \times 10^{-7}$ & $18.97 \times 10^{-7}$ & 0.119 \\
$c_{3}$ & -5.32 & $68.77 \times 10^{-8}$ & $96.96 \times 10^{-8}$ & 0.013 \\
$c_{4}$ & -5.35 & $35.74 \times 10^{-8}$ & $10.79 \times 10^{-7}$ & 0.019 \\
$c_{5}$ & -10.98 & $25.29 \times 10^{-7}$ & $15.92 \times 10^{-7}$ & 0.079 \\
$c_{6}$ & 5.62 & $38.83 \times 10^{-10}$ & $-76.75 \times 10^{-8}$ & 0.006 \\
$c_{7}$ & -5.74 & $10.30 \times 10^{-7}$ & $10.26 \times 10^{-7}$ & 0.013 \\
$c_{8}$ & 3.94 & $42.30 \times 10^{-8}$ & $-67.14 \times 10^{-8}$ & 0.007 \\
$c_{9}$ & 0.25 & $24.73 \times 10^{-8}$ & $24.87 \times 10^{-9}$ & 0.002 \\
\hline
\end{tabular}

TABlE 5. Parameters estimated at the Palanga station with the corresponding values of the standard errors and values obtained from the spatial models.

\begin{tabular}{rrrrrr}
\hline & $a_{1}^{i}$ & $b_{1}^{i}$ & $b_{2}^{i}$ & $b_{3}^{i}$ & $\alpha_{i}$ \\
\hline Estimated & 7.32 & -0.18 & 9.86 & 51.06 & 0.81 \\
Std. error & 0.24 & 0.06 & 0.08 & 0.48 & 0.01 \\
Fitted & 6.23 & -0.07 & 10.50 & 47.96 & 0.83 \\
\hline & $c_{1}^{i}$ & $c_{2}^{i}$ & $c_{3}^{i}$ & $c_{4}^{i}$ & $c_{5}^{i}$ \\
\hline Estimated & 5.01 & 2.18 & 0.65 & 1.24 & -1.34 \\
Std. error & 0.15 & 0.21 & 0.21 & 0.21 & 0.21 \\
Fitted & 4.85 & 2.35 & 0.92 & 1.46 & -0.29 \\
\hline & $c_{6}^{i}$ & $c_{7}^{i}$ & $c_{8}^{i}$ & $c_{9}^{i}$ & \\
\hline Estimated & 1.19 & 0.62 & -0.10 & 0.66 & \\
Std. error & 0.21 & 0.21 & 0.21 & 0.21 & \\
Fitted & 0.85 & 0.96 & -0.09 & 0.49 & \\
\hline
\end{tabular}


TABle 6. Parameters estimated at the Traku Voke station with the corresponding values of the standard errors and values obtained from the spatial models.

\begin{tabular}{rrrrrr}
\hline & $a_{1}^{i}$ & $b_{1}^{i}$ & $b_{2}^{i}$ & $b_{3}^{i}$ & $\alpha_{i}$ \\
\hline Estimated & 5.99 & -0.10 & 11.30 & 48.41 & 0.82 \\
Std. error & 0.16 & 0.04 & 0.05 & 0.28 & 0.00 \\
Fitted & 5.85 & -0.16 & 11.27 & 44.79 & 0.81 \\
\hline & $c_{1}^{i}$ & $c_{2}^{i}$ & $c_{3}^{i}$ & $c_{4}^{i}$ & $c_{5}^{i}$ \\
\hline Estimated & 5.95 & 2.25 & 0.99 & 1.59 & -0.16 \\
Std. error & 0.13 & 0.18 & 0.18 & 0.18 & 0.18 \\
Fitted & 6.27 & 3.47 & 0.95 & 1.39 & 0.10 \\
\hline & $c_{6}^{i}$ & $c_{7}^{i}$ & $c_{8}^{i}$ & $c_{9}^{i}$ & \\
\hline Estimated & 0.64 & 0.93 & 0.23 & 0.36 & \\
Std. error & 0.18 & 0.18 & 0.18 & 0.18 & \\
Fitted & 0.97 & 1.06 & 0.12 & 0.54 & \\
\hline
\end{tabular}

